Article

\title{
Analyses of the Association between Thyroid Cancer and Osteoporosis/Fracture Histories: A Cross-Sectional Study Using KoGES HEXA Data
}

\author{
Young-Ju Jin ${ }^{1}$, Chang-Myeon Song ${ }^{2}$ (D), Bum-Jung Park ${ }^{3}$ and Hyo-Geun Choi ${ }^{3,4, *}$ \\ 1 Department of Otorhinolaryngology-Head and Neck Surgery, Wonkwang University Hospital, \\ Wonkwang University College of Medicine, Iksan 54538, Korea; chindol1@wku.ac.kr \\ 2 Department of Otolaryngology-Head and Neck Surgery, Hanyang University College of Medicine, \\ Seoul 04763, Korea; cmsong@hanyang.ac.kr \\ 3 Department of Otorhinolaryngology-Head and Neck Surgery, Hallym University College of Medicine, \\ Anyang 14103, Korea; pbj426@hallym.ac.kr \\ 4 Hallym Data Science Laboratory, Hallym University College of Medicine, Anyang 14103, Korea \\ * Correspondence: hgchoi@hallym.ac.kr; Tel.: +82-31-380-3849; Fax: +82-31-386-3860
}

Citation: Jin, Y.-J.; Song, C.-M.; Park, B.-J.; Choi, H.-G. Analyses of the Association between Thyroid Cancer and Osteoporosis/Fracture Histories: A Cross-Sectional Study Using KoGES HEXA Data. Int. J. Environ. Res. Public Health 2021, 18, 4732. https://doi.org/10.3390/ijerph18094732

Academic Editor: Paul B. Tchounwou

Received: 9 March 2021

Accepted: 28 April 2021

Published: 29 April 2021

Publisher's Note: MDPI stays neutral with regard to jurisdictional claims in published maps and institutional affiliations.

Copyright: (c) 2021 by the authors. Licensee MDPI, Basel, Switzerland. This article is an open access article distributed under the terms and conditions of the Creative Commons Attribution (CC BY) license (https:// creativecommons.org/licenses/by/ $4.0 /)$.

\begin{abstract}
Background: The purpose of this study was to determine the association among thyroid cancer, osteoporosis and fracture history. (2) Methods: The data collected from 2004 through 2016 for the Korean Genome and Epidemiology Study were retrieved. For a total of 1349 participants with thyroid cancer and 163,629 control participants, the odds ratios (ORs) with $95 \%$ confidence intervals (CIs) of osteoporosis and fracture history were evaluated using a logistic regression model. (3) Results: The adjusted ORs of osteoporosis comparing thyroid cancer with the control group were $1.41(95 \% \mathrm{CI}=1.18-1.70, p<0.001)$ for all participants and $1.43(95 \% \mathrm{CI}=1.19-1.71, p<0.001)$ for women. The adjusted ORs of fracture history comparing these two groups were not significantly associated within the entire group of participants, men only or women only. (4) Conclusions: The adjusted OR of osteoporosis was significantly higher than 1, comparing thyroid cancer with the control group, especially in women. The adjusted OR of fractures was not significantly higher than 1 , comparing the thyroid cancer group with the control group.
\end{abstract}

Keywords: thyroid cancer; osteoporosis; fracture; cross-sectional study

\section{Introduction}

Osteoporosis is a bone disease that is characterized by the loss of bone mass and strength, which lead to fragility fractures and thus severe morbidities and mortality. The prevalence of osteoporosis and osteopenia in the US is 35.5 million in women and 18.2 million in men [1]. Moreover, approximately $50 \%$ of females and $20 \%$ of males experience fragility fractures of the hip, wrist or spine in their aged life [2]. In Korea, the prevalence of osteoporosis was reported to be $38.0 \%$ in females and $7.3 \%$ in males aged over 50 years old [3]. The main causes of osteoporosis are related to age, calcium deficiency, vitamin D deficiency, secondary hyperparathyroidism, inflammatory diseases such as rheumatoid arthritis, glucocorticoid use, excessive alcohol use and estrogen deficiency, especially in postmenopausal women $[4,5]$.

Thyroid cancer is the most common endocrine malignancy. The incidence of thyroid cancer increased 3.6\% per year during 1974-2013 in a US study [6]. The incidence of thyroid cancer increased 22.6\% during 1999-2011 in Korea [7]. Regarding treatment, various kinds of thyroidectomy, including partial, subtotal or total thyroidectomy, are performed and followed with radioactive iodine (RAI) therapy after thyrotropin (TSH) suppression.

Previous studies have suggested that the incidence of osteoporosis is higher in patients with than in those without thyroid cancer [8-11]. In a population-based observational cohort study in Taiwan, the total thyroidectomy group showed a significantly elevated 
long-term risk of osteoporosis [12]. Although osteoporosis causes fragility fractures, the relationship between thyroid cancer and fracture history remains unclear. Some studies have suggested that the risk of fracture is significantly increased in thyroid cancer patients $[13,14]$. However, other studies have suggested that the risk of fracture is not higher in thyroid cancer or total thyroidectomy patients than in patients who have not undergone thyroidectomy $[9,12]$. Moreover, patients with TSH suppression therapy but not those with thyroid cancer experience a high number of fractures [15-17]. Therefore, we compared the prevalence of fractures as well as osteoporosis between thyroid cancer patients and controls.

The aim of this study was to evaluate the association among thyroid cancer, osteoporosis and fractures based on Korean Genome and Epidemiology Study (KoGES) health examinee (HEXA) data. The odds ratio itself compares these two groups.

\section{Materials and Methods}

\subsection{Study Population and Data Collection}

The ethics committee of Hallym University (20 February 2019) approved the use of these data. The requirement for written informed consent was waived by the institutional review board. This prospective cohort study used data collected from 2004 through 2016 for the Korean Genome and Epidemiology Study (KoGES). A detailed description of these data is provided in a previous study [18]. Among the KoGES consortium data, we used KoGES health examinee (HEXA) data for urban residence participants $\geq 40$ years old.

\subsection{Participants Selection}

Among 173,209 participants, we excluded participants who lacked data on height or weight $(n=934)$; smoking history $(n=1390)$; alcohol consumption habits $(n=331)$; hypertension, diabetes mellitus, or dyslipidemia histories $(n=203)$; arthritis or thyroid disease histories $(n=60)$; and osteoporosis history $(n=37)$. Among the thyroid cancer participants, we excluded those who previously had other types of cancer (gastric cancer, hepatic cancer, colon cancer, breast cancer, lung cancer, uterine cervix cancer, or bladder cancer, $n=83$ ). Among the control participants without a history of thyroid cancer, participants with a history of other cancers were excluded $(n=5193)$. Finally, 1349 thyroid cancer and 163,629 control (no history of thyroid cancer) participants were selected (Figure 1). Then, we analyzed the histories of osteoporosis between the thyroid cancer and control participants (primary objective).

Then, we analyzed the histories of any fractures between the thyroid cancer and control participants (secondary objective). In this study, we excluded 64 participants with thyroid cancer and 35,957 control participants who did not have data on their fracture history.

\subsection{Survey}

The participants were asked whether they previously had cancer, including thyroid cancer, gastric cancer, hepatic cancer, colon cancer, breast cancer, lung cancer, cervical cancer, or bladder cancer, by trained interviewers. They were also asked about their past medical history regarding hypertension, diabetes mellitus, dyslipidemia, arthritis, and thyroid diseases (hyperthyroidism or hypothyroidism). Body mass index (BMI) was calculated in $\mathrm{kg} / \mathrm{m}^{2}$ using the health checkup data. The patients were categorized as nonsmokers (smoked $<100$ cigarettes in their entire life), past smokers (quit more than one year ago), or current smokers. According to their alcohol consumption habits, the patients were categorized as non-drinkers (no history of alcohol consumption in their entire life), past drinkers (quit alcohol consumption), or current drinkers. The participants were asked whether they had been diagnosed with osteoporosis or any fractures. 


\section{3,209 participants from KoGES HEXA study from 2004 through 2016}

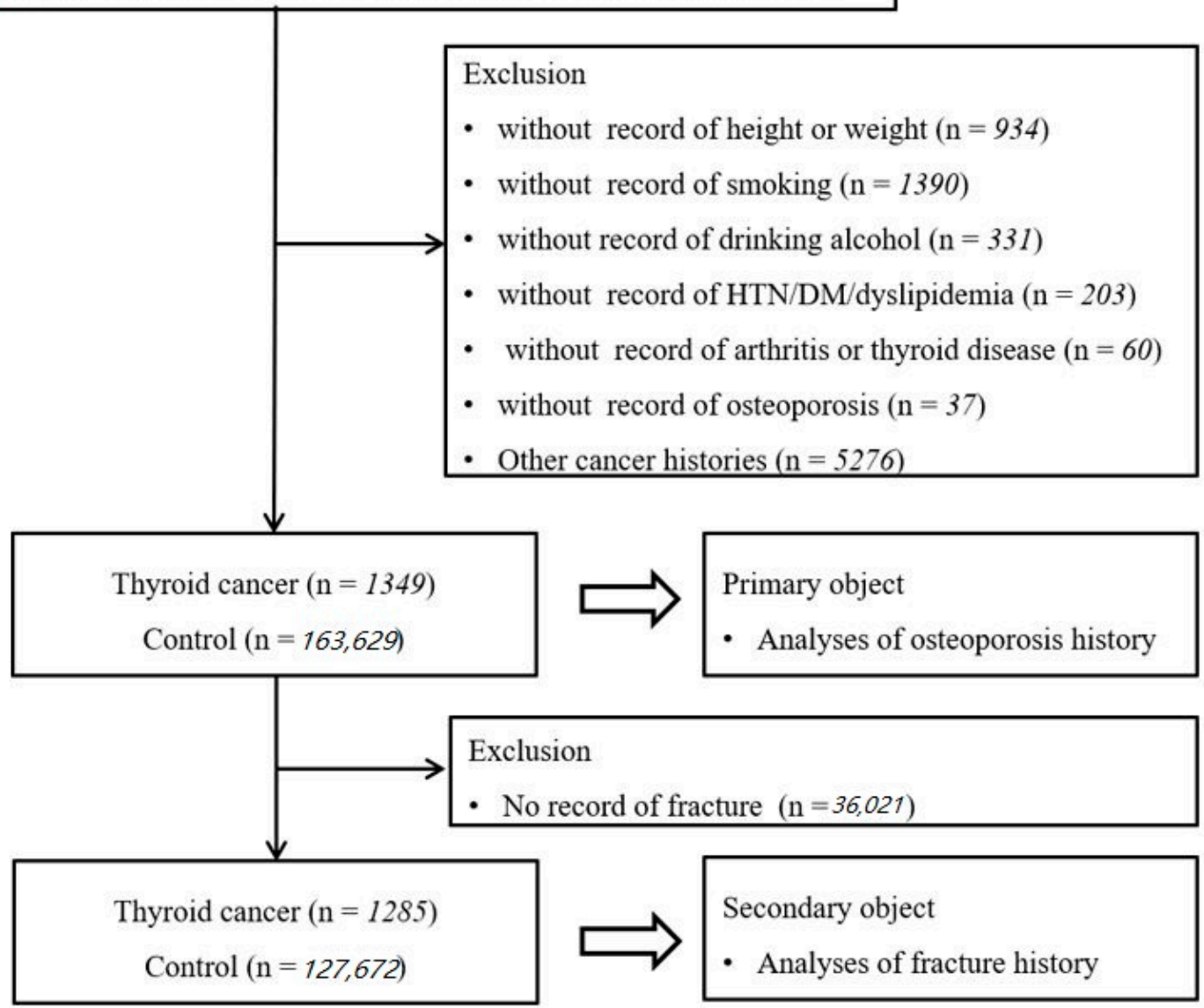

Figure 1. A schematic illustration of the participant selection process that was used in the present study. Of a total of 173,209 participants, 1349 thyroid cancer and 163,629 control (non-thyroid cancer) participants were selected.

\subsection{Statistical Analyses}

The chi-square test was used to compare the rates of each sex, hypertension, diabetes mellitus, dyslipidemia, arthritis, any thyroid disease, smoking, alcohol consumption, and osteoporosis. Independent $t$-tests were used to compare age and BMI.

To analyze the OR of thyroid cancer for osteoporosis, a logistic regression model was used. For the primary objective, crude and adjusted models (age, sex, BMI, hypertension, diabetes mellitus, dyslipidemia, arthritis, thyroid disease histories, smoking, and alcohol consumption) were used. For the secondary objective, crude and adjusted models (previous adjusted model plus osteoporosis histories) were used.

Two-tailed analyses were conducted, and $p$ values less than 0.05 were considered to indicate significance. The results were statistically analyzed using SPSS, version 24.0 (IBM, Armonk, NY, USA).

\section{Results}

\subsection{Detailed Descriptions}

\subsubsection{General Characteristics of Participants}

The proportions of women ( $91.5 \%$ vs. $65.4 \%)$, patients with thyroid disease $(18.8 \%$ vs. $4.5 \%)$, non-smokers $(92.7 \%$ vs. $72.7 \%)$, non-drinkers ( $66.8 \%$ vs. $50.4 \%)$ and patients with osteoporosis ( $11.0 \%$ vs. $6.7 \%$ ) were higher in the thyroid cancer group than in the control group with statistical significance. On the other hand, the proportions of past smokers 
(4.5\% vs. $14.7 \%)$, current smokers ( $2.7 \%$ vs. $12.7 \%)$, past drinkers $(3.0 \%$ vs. $3.8 \%)$ and current drinkers (30.2\% vs. $45.8 \%$ ) were lower in the thyroid cancer group than in the control group. The mean age was lower ( 52.3 years vs. 53.0 years) in the thyroid cancer group compared with the control group with statistical significance (Table 1).

Table 1. General characteristics of participants.

\begin{tabular}{lccc}
\hline \multirow{2}{*}{ Characteristics } & \multicolumn{2}{c}{ Thyroid Cancer Histories } & $p$-Value \\
\cline { 2 - 4 } & Yes & No & \\
\hline Number $(n, \%)$ & $1349(100.0)$ & $163,629(100.0)$ & $<0.001^{*}$ \\
Age $(y$, mean, SD) & $52.3(7.3)$ & $53.0(8.4)$ & $<0.001^{*}$ \\
Sex (women, $n, \%)$ & $1235(91.5)$ & $106,993(65.4)$ & 0.646 \\
BMI $\left(\mathrm{kg} / \mathrm{m}^{2}\right.$, mean, SD) & $23.9(2.9)$ & $23.9(2.9)$ & 0.555 \\
Hypertension $(n, \%)$ & $270(20.0)$ & $31,706(19.4)$ & $0.006^{*}$ \\
Diabetes mellitus $(n, \%)$ & $64(4.7)$ & $10,833(6.6)$ & $0.038^{*}$ \\
Dyslipidemia $(n, \%)$ & $144(10.7)$ & $14,800(9.0)$ & 0.659 \\
Arthritis $(n, \%)$ & $124(9.2)$ & $15,621(9.5)$ & $<0.001^{*}$ \\
Thyroid diseases $(n, \%)$ & $253(18.8)$ & $7365(4.5)$ & \\
Smoking $(n, \%)$ & & & \\
$\quad$ Non-smoker & $1251(92.7)$ & $118,894(72.7)$ & $<001^{*}$ \\
$\quad$ Past smoker & $61(4.5)$ & $23,973(14.7)$ & \\
Current smoker & $37(2.7)$ & $20,762(12.7)$ & \\
Drinking alcohol $(n, \%)$ & & & \\
$\quad$ Non-drinker & $901(66.8)$ & $62,443(50.4)$ & $<0.001^{*}$ \\
$\quad$ Past drinker & $41(3.0)$ & $74,949(45.8)$ & \\
$\quad$ Current drinker & $407(30.2)$ & $10,939(6.7)$ & \\
Osteoporosis $(n, \%)$ & $149(11.0)$ & & \\
\hline
\end{tabular}

${ }^{*}$ Independent $t$-test or Chi-square test, Statistical significance at $p<0.05$.

\subsubsection{ORs of Osteoporosis Comparing Participants with Thyroid Cancer to Control}

The adjusted ORs of osteoporosis comparing participants with thyroid cancer to control were $1.41(95 \% \mathrm{CI}=1.18-1.70, p<0.001)$ in all participants and $1.43(95 \% \mathrm{CI}=1.19-1.71$, $p<0.001$ ) in women (Table 2). On the other hand, a statistical difference was not present between the control group and men.

Table 2. Odds ratios of osteoporosis comparing participants with thyroid cancer to control.

\begin{tabular}{|c|c|c|c|c|c|}
\hline \multirow{2}{*}{ Variables } & \multirow{2}{*}{ Osteoporosis/Participants $(n, \%)$} & \multicolumn{4}{|c|}{ ORs for Osteoporosis } \\
\hline & & Crude & $p$-Value & Adjusted $1^{\dagger}$ & $p$-Value \\
\hline \multicolumn{6}{|l|}{ Total participants $(n=164,978)$} \\
\hline Thyroid cancer & $149 / 1349(11.0)$ & $1.73(1.46-2.06)$ & $<0.001 *$ & $1.41(1.18-1.70)$ & $<0.001$ * \\
\hline Control & $10,939 / 163,629(6.7)$ & 1 & & 1 & \\
\hline \multicolumn{6}{|l|}{ Men $(n=56,750)$} \\
\hline Thyroid cancer & $1 / 114(0.9)$ & $1.05(0.15-7.54)$ & 0.961 & $0.98(0.14-7.07)$ & 0.982 \\
\hline Control & $473 / 56,636(0.8)$ & 1 & & 1 & \\
\hline \multicolumn{6}{|l|}{ Women $(n=108,228)$} \\
\hline Thyroid cancer & $149 / 1235(12.0)$ & $1.26(1.06-1.49)$ & $0.010 *$ & $1.43(1.19-1.71)$ & $<0.001$ * \\
\hline Control & $10,466 / 106,993(9.8)$ & 1 & & 1 & \\
\hline
\end{tabular}

${ }^{*}$ Logistic regression was analyzed, Significance at $p<0.05 ;{ }^{\dagger}$ It was adjusted for age, sex, body mass index (BMI), hypertension, diabetes mellitus, dyslipidemia, arthritis, thyroid disease histories, smoking, and drinking alcohol.

\subsubsection{ORs of Fracture Histories Comparing Participants with Thyroid Cancer to Control}

The adjusted ORs of thyroid cancer for fracture history were not statistically significant or larger than 1 in the entire group of participants, men only or women only (Table 3). 
Table 3. Odds ratios of fracture histories comparing participants with thyroid cancer to control.

\begin{tabular}{|c|c|c|c|c|c|}
\hline \multirow{2}{*}{ Variables } & \multirow{2}{*}{ Fracture/Participants $(n, \%)$} & \multicolumn{4}{|c|}{ ORs for Fracture } \\
\hline & & Crude & $p$-Value & Adjusted $1^{+}$ & $p$-Value \\
\hline \multicolumn{6}{|l|}{ Total participants $(n=128,957)$} \\
\hline Thyroid cancer & $150 / 1285$ (11.7) & $0.92(0.77-1.09)$ & 0.307 & $1.00(0.84-1.19)$ & 0.982 \\
\hline Control & $16,119 / 127,672(12.6)$ & 1 & & 1 & \\
\hline \multicolumn{6}{|l|}{ Men $(n=45,162)$} \\
\hline Thyroid cancer & $12 / 109(11.0)$ & $0.70(0.39-1.28)$ & 0.252 & $0.74(0.41-1.35)$ & 0.324 \\
\hline Control & $6734 / 45,053(14.9)$ & 1 & & 1 & \\
\hline \multicolumn{6}{|l|}{ Women $(n=83,795)$} \\
\hline Thyroid cancer & 138/1176 (11.7) & $1.04(0.87-1.24)$ & 0.687 & $1.06(0.88-1.27)$ & 0.528 \\
\hline Control & $9385 / 82,619(11.4)$ & 1 & & 1 & \\
\hline
\end{tabular}

${ }^{\dagger}$ It was adjusted for age, sex, body mass index (BMI), hypertension, diabetes mellitus, dyslipidemia, arthritis, thyroid disease histories, smoking, drinking alcohol, and osteoporosis.

\section{Discussion}

In our study, the ORs of osteoporosis were significantly higher than 1, comparing participants with thyroid cancer with control, especially in women over 40 years old. Fracture history did not significantly differ between the thyroid cancer group and the control group.

In accordance with the results of our study, in previous studies, the risk of osteoporosis was significantly higher in the thyroid cancer group than in the control group. In a large case-control study in the US $(n=10,370)$, osteoporosis was more common in the thyroid cancer group than in the control group (7.3\% vs. 5.3\%) [9]. In a population-based US study $(n=3706)$, the HRs of osteoporosis were higher $1-5$ years after thyroid cancer diagnoses and $5-10$ years after thyroid cancer diagnoses in both the age groups $<40$ years and $>40$ years than in the control group [8]. In a small Japanese case-control study $(n=35)$, the prevalence of severe osteoporosis was significantly higher in the papillary thyroid carcinoma group than in the control group $(33.3 \%$ vs. $11.4 \%, p<0.05)$ [10]. Possible causes for the association between thyroid cancer and osteoporosis are listed below. First, TSH suppression therapy and hyperthyroidism may be risk factors for osteoporosis, as these factors enhance osteoclastic bone resorption in adults [19]. Levothyroxine replacement has been prescribed after total thyroidectomy to suppress TSH and supply thyroid hormones. TSH is suggested to be a key negative regulator of bone turnover. When it was suppressed below $0.4 \mathrm{mIU} / \mathrm{L}$, the risk of osteoporosis increases in differentiated thyroid cancer patients [20]. TSH suppression and an older age have a synergistic effect on the reduction of bone marrow density [21]. Second, vitamin D deficiency may be a common risk factor for thyroid cancer and osteoporosis [22]. The major functions of vitamin D are to maintain homeostasis regarding the levels of calcium and phosphorus in the body and to preserve bone health. Moreover, vitamin D deficiency has been reported to be positively associated with thyroid cancer through the increased expression of p27, known as a tumor suppressor protein, by binding to the vitamin D receptor [23-25]. Third, autoimmunity may link thyroid cancer and osteoporosis by activating bone resorption. PTC patients with severe osteoporosis have a higher prevalence of TPO-Ab than patients without severe osteoporosis [10]. The presence of TPO-Ab is associated with osteoporotic fractures in euthyroid postmenopausal women [26]. On the other hand, the risk of thyroid cancer is not related to TPO-Ab levels but is associated with Tg-Ab levels [27]. However, the associations between autoimmune diseases and thyroid cancer are still controversial. Fourth, calcitonin deficiency after total thyroidectomy is a possible cause of osteoporosis. Calcitonin is secreted in C cells of the thyroid gland and acts as a potent inhibitor of osteoclastic bone resorption to maintain stable serum calcium levels [28,29]. However, the physiological action of calcitonin on bone tissue is not fully understood, although this hormone has been approved for the treatment of osteoporosis for approximately 30 years [30]. Fifth, the incidence rates of both thyroid cancer and osteoporosis are much higher in women than 
in men [31,32]. Therefore, the levels of female sex hormones, such as estrogen, could be related to the etiology of these two diseases. However, additional studies are needed on this topic.

The OR of fractures history was not significantly higher than 1 , comparing participants with thyroid cancer with the control group. Few studies on the association between thyroid cancer and osteoporotic fractures have been conducted. In a previous study, a history of thyroidectomy did not increase the overall fracture risk in 630 women with respect to the community incidence rate [13]. In a large-scale case-control US study $(n=10,370)$, thyroid cancer was not related to an increased risk of fracture [9]. In a large population-based Korean study $(n=185,956)$, both high and low doses of levothyroxine treatment were linked to a higher risk of fracture in post thyroidectomy patients [17]. However, the association between hypo- or hyperthyroidism and fracture history was not decisive. In another study, low-dose levothyroxine was not associated with fracture risk, and antithyroid drugs significantly reduced the risk of fractures [16]. Thyroid cancer patients may be more frequently followed and actively treated with osteoporosis medication than controls. However, this topic needs to be studied further.

In our subgroup analysis, the ORs for osteoporosis were significantly higher in women with thyroid cancer only than in women in the control group. The incidence rates of osteoporosis and thyroid cancer were much higher in women than in men. However, the risk of osteoporosis was higher in the postmenopausal stage due to estrogen deficiency, and thyroid cancer was more common in premenopausal women than in postmenopausal women [33,34]. Therefore, additional studies need to be conducted to determine the mechanism of the link between female hormone levels and these two diseases. The cause of the inconsistency in the results between the men and women could be related to the sex differences in thyroid cancer incidence. The number of males with thyroid cancer was too small in our study, although to the total participant number is considered large. In our study, the total number of thyroid cancer patients was 1349, and 1235 patients (91.5\%) were women. Therefore, the number of men $(n=114)$ may have been too small to detect an association between thyroid cancer and osteoporosis. A previous large populationbased cohort study in Taiwan showed similar results. Among 1426 thyroid cancer patients, the women $(n=1208)$ showed a significantly increased incidence of osteoporosis after thyroid operation compared with the controls. However, the men $(n=218)$ did not show statistically significantly different results compared with the controls [12]. Moreover, in a US veteran study including 8689 male patients, osteoporosis was more common in male thyroid cancer patients than in the controls [9].

There are several strengths of this study. First, we adjusted for the possible risk factors of thyroid cancer or osteoporosis to minimize the effects of confounding factors such as BMI, aging, diabetes mellitus, a history of thyroid disease, smoking and alcohol consumption. Second, the models were adjusted to account for a history of arthritis because autoantibodies against RA, systemic inflammatory conditions and glucocorticoid use can increase the prevalence of osteoporosis [35]. Third, this was a large-scale population-based prospective study. There are several limitations. First, recall bias may have been present in this study because information about previous diseases and possible risk factors for osteoporosis were reported by the patients rather than retrieved from medical records. Second, osteoporosis was not diagnosed using bone mineral density measurements, as recommended by the WHO. Third, this database does not contain medication information, and medications such as steroids, diuretics and thyroxine can influence the incidence of osteoporosis. Fourth, the results of thyroid function tests, such as TSH, free T4, TPO-Ab and $\mathrm{Ag}-\mathrm{Ab}$ tests, were not available, and thyroid function may influence the prevalence of osteoporosis. Fifth, specific thyroid cancer treatments were not investigated in this study, such as thyroid operations and RAI treatment. Sixth, this study has limitations as a cross-sectional study because we did not have the information as to when participants were diagnosed with thyroid cancer and fractures or osteoporosis. Seventh, we cannot evaluate according to thyroid cancer type, grade and stage due to lack of information. However, 
papillary thyroid carcinoma accounted for $97.2 \%$ of Korean thyroid cancer according to the previous national epidemiologic study [36].

\section{Conclusions}

Our study provides very important evidence of the relationship between thyroid cancer and the risk of osteoporosis. The adjusted OR of osteoporosis was significantly higher than 1, comparing the thyroid cancer group with the control group, especially in the female group. The adjusted OR of fractures was not significantly higher than 1, comparing the thyroid cancer group with the control groups. Additional studies are needed to determine the effect of thyroid-stimulating hormones, including free T4, TPO-Ab, Ag- $\mathrm{Ab}$ and estrogen.

Author Contributions: Conceptualization, B.-J.P. and H.-G.C.; investigation, C.-M.S., Y.-J.J.; data curation, B.-J.P., H.-G.C., C.-M.S.; formal analysis, H.-G.C., Y.-J.J.; supervision, B.-J.P. and H.-G.C.; validation, Y.-J.J. and H.-G.C.; original draft writing, C.-M.S., Y.-J.J.; review and editing, C.-M.S., Y.-J.J. and H.-G.C.; project administration, B.-J.P. and H.-G.C.; funding acquisition, H.-G.C. All authors have read and agreed to the published version of the manuscript.

Funding: This research was funded by a research grant (NRF-2018-R1D1A1A0-2085328 and NRF2019-R1G1A1-099842) from the National Research Foundation (NRF) of Korea and Hallym University Research Fund, grant number HURF-2020-63.

Institutional Review Board Statement: The ethics committee of Hallym University (2019-02-020) approved the use of these data.

Informed Consent Statement: The requirement for written informed consent was waived by the institutional review board.

Data Availability Statement: Release of the data by the authors is not legally allowed. All of the data are available on the database of the Korean genome and epidemiology study (KoGES) health examinee (HEXA) data in National Research Institute of Health (NIH) https: / /nih.go.kr/ (accessed on 3 November 2020). NIH permits access to all of these data via download for any researcher who promises to follow the research ethics.

Acknowledgments: The manuscript was edited for proper English language, grammar, punctuation, spelling, and overall style by the highly qualified native English-speaking editors.

Conflicts of Interest: The authors declare no conflict of interest.

\section{References}

1. Goode, S.C.; Wright, T.F.; Lynch, C. Osteoporosis screening and treatment: A collaborative approach. J. Nurse Pract. 2020, 16, 60-63. [CrossRef]

2. Friedman, S.M.; Mendelson, D.A. Epidemiology of fragility fractures. Clin. Geriatr. Med. 2014, 30, 175-181. [CrossRef] [PubMed]

3. Park, E.J.; Joo, I.W.; Jang, M.-J.; Kim, Y.T.; Oh, K.; Oh, H.J. Prevalence of osteoporosis in the Korean population based on Korea National Health and Nutrition Examination Survey (KNHANES), 2008-2011. Yonsei Med. J. 2014, 55, 1049-1057. [CrossRef] [PubMed]

4. Raisz, L.G. Pathogenesis of osteoporosis: Concepts, conflicts, and prospects. J. Clin. Investig. 2005, 115, 3318-3325. [CrossRef]

5. Tu, K.N.; Lie, J.D.; Wan, C.K.V.; Cameron, M.; Austel, A.G.; Nguyen, J.K.; Van, K.; Hyun, D. Osteoporosis: A review of treatment options. Pharm. Ther. 2018, 43, 92.

6. Lim, H.; Devesa, S.S.; Sosa, J.A.; Check, D.; Kitahara, C.M. Trends in thyroid cancer incidence and mortality in the United States, 1974-2013. JAMA 2017, 317, 1338-1348. [CrossRef]

7. Jung, K.-W.; Won, Y.-J.; Kong, H.-J.; Lee, E.S. Cancer statistics in Korea: Incidence, mortality, survival, and prevalence in 2016. Cancer Res. Treat. 2019, 51, 417. [CrossRef]

8. Blackburn, B.E.; Ganz, P.A.; Rowe, K.; Snyder, J.; Wan, Y.; Deshmukh, V.; Newman, M.; Fraser, A.; Smith, K.; Herget, K. Aging-related disease risks among young thyroid cancer survivors. Cancer Epidemiol. Prev. Biomark. 2017, 26, 1695-1704. [CrossRef]

9. Papaleontiou, M.; Banerjee, M.; Reyes-Gastelum, D.; Hawley, S.T.; Haymart, M.R. Risk of Osteoporosis and Fractures in Patients with Thyroid Cancer: A Case-Control Study in US Veterans. Oncologist 2019, 24, 1166. [CrossRef]

10. Notsu, M.; Yamauchi, M.; Morita, M.; Nawata, K.; Sugimoto, T. Papillary thyroid carcinoma is a risk factor for severe osteoporosis. J. Bone Miner. Metab. 2020, 38, 264-270. [CrossRef] 
11. Lin, S.-Y.; Lin, C.-L.; Chen, H.-T.; Kao, C.-H. Risk of osteoporosis in thyroid cancer patients using levothyroxine: A populationbased study. Curr. Med. Res. Opin. 2018, 34, 805-812. [CrossRef] [PubMed]

12. Hung, C.-L.; Yeh, C.-C.; Sung, P.-S.; Hung, C.-J.; Muo, C.-H.; Sung, F.-C.; Jou, I.-M.; Tsai, K.-J. Is partial or total thyroidectomy associated with risk of long-term osteoporosis: A nationwide population-based study. World J. Surg. 2018, 42, $2864-2871$. [CrossRef] [PubMed]

13. Melton, L., III; Ardila, E.; Crowson, C.; O'Fallon, W.; Khosla, S. Fractures following thyroidectomy in women: A population-based cohort study. Bone 2000, 27, 695-700. [CrossRef]

14. Nguyen, T.T.; Heath, H., III; Bryant, S.C.; O’Fallon, W.M.; Melton, L.J., III. Fractures After Thyroidectomy in Men: A PopulationBased Cohort Study. J. Bone Miner. Res. 1997, 12, 1092-1099. [CrossRef] [PubMed]

15. Lee, Y.; Yoon, B.-H.; Lee, S.; Chung, Y.K.; Lee, Y.-K. Risk of Osteoporotic Fractures after Thyroid-stimulating Hormone Suppression Therapy in Patients with Thyroid Cancer. J. Bone Metab. 2019, 26, 45-50. [CrossRef]

16. Vestergaard, P.; Rejnmark, L.; Mosekilde, L. Influence of hyper-and hypothyroidism, and the effects of treatment with antithyroid drugs and levothyroxine on fracture risk. Calcif. Tissue Int. 2005, 77, 139-144. [CrossRef] [PubMed]

17. Shin, D.W.; Suh, B.; Lim, H.; Yun, J.M.; Song, S.O.; Park, Y. J-shaped association between postoperative levothyroxine dosage and fracture risk in thyroid cancer patients: A retrospective cohort study. J. Bone Miner. Res. 2018, 33, 1037-1043. [CrossRef]

18. Kim, Y.; Han, B.-G.; Group, K. Cohort profile: The Korean genome and epidemiology study (KoGES) consortium. Int. J. Epidemiol. 2017, 46, e20. [CrossRef]

19. Nicholls, J.J.; Brassill, M.J.; Williams, G.R.; Bassett, J. The skeletal consequences of thyrotoxicosis. J. Endocrinol. 2012, $213,209-221$. [CrossRef]

20. Wang, L.Y.; Smith, A.W.; Palmer, F.L.; Tuttle, R.M.; Mahrous, A.; Nixon, I.J.; Patel, S.G.; Ganly, I.; Fagin, J.A.; Boucai, L. Thyrotropin suppression increases the risk of osteoporosis without decreasing recurrence in ATA low-and intermediate-risk patients with differentiated thyroid carcinoma. Thyroid 2015, 25, 300-307. [CrossRef]

21. Sugitani, I.; Fujimoto, Y. Effect of postoperative thyrotropin suppressive therapy on bone mineral density in patients with papillary thyroid carcinoma: A prospective controlled study. Surgery 2011, 150, 1250-1257. [CrossRef] [PubMed]

22. Muscogiuri, G.; Tirabassi, G.; Bizzaro, G.; Orio, F.; Paschou, S.; Vryonidou, A.; Balercia, G.; Shoenfeld, Y.; Colao, A. Vitamin D and thyroid disease: To D or not to D? Eur. J. Clin. Nutr. 2015, 69, 291-296. [CrossRef] [PubMed]

23. Basit, S. Vitamin D in health and disease: A literature review. Br. J. Biomed. Sci. 2013, 70, 161-172. [CrossRef]

24. Ahn, H.Y.; Chung, Y.J.; Park, K.-Y.; Cho, B.Y. Serum 25-hydroxyvitamin D level does not affect the aggressiveness and prognosis of papillary thyroid cancer. Thyroid 2016, 26, 429-433. [CrossRef]

25. Zhao, J.; Wang, H.; Zhang, Z.; Zhou, X.; Yao, J.; Zhang, R.; Liao, L.; Dong, J. Vitamin D deficiency as a risk factor for thyroid cancer: A meta-analysis of case-control studies. Nutrition 2019, 57, 5-11. [CrossRef]

26. Polovina, S.P.; Miljic, D.; Zivojinovic, S.; Milic, N.; Micic, D.; Brkic, V.P. The impact of thyroid autoimmunity (TPOAb) on bone density and fracture risk in postmenopausal women. Hormones 2017, 16, 54-61. [PubMed]

27. Azizi, G.; Malchoff, C. Autoimmune thyroid disease: A risk factor for thyroid cancer. Endocr. Pract. 2011, 17, 201-209. [CrossRef] [PubMed]

28. Wimalawansa, S.J. Physiology of Calcitonin and Its Therapeutic Uses. In Encyclopedia Endocrine Diseases, 2nd ed.; Elsevier: Amsterdam, The Netherlands, 2018; Volume 4, pp. 178-191.

29. Davey, R.A.; Findlay, D.M. Calcitonin: Physiology or fantasy? J. Bone Miner. Res. 2013, 28, 973-979. [CrossRef] [PubMed]

30. De Paula, F.; Rosen, C. Back to the future: Revisiting parathyroid hormone and calcitonin control of bone remodeling. Horm. Metab. Res. 2010, 42, 299-306. [CrossRef]

31. Burge, R.; Dawson-Hughes, B.; Solomon, D.H.; Wong, J.B.; King, A.; Tosteson, A. Incidence and economic burden of osteoporosisrelated fractures in the United States, 2005-2025. J. Bone Miner. Res. 2007, 22, 465-475. [CrossRef]

32. Cao, Y.; Wang, Z.; Gu, J.; Hu, F.; Qi, Y.; Yin, Q.; Sun, Q.; Li, G.; Quan, B. Reproductive factors but not hormonal factors associated with thyroid cancer risk: A systematic review and meta-analysis. Biomed. Res. Int. 2015, 2015, 103515. [CrossRef] [PubMed]

33. Holm, J.P.; Hyldstrup, L.; Jensen, J.-E.B. Time trends in osteoporosis risk factor profiles: A comparative analysis of risk factors, comorbidities, and medications over twelve years. Endocrine 2016, 54, 241-255. [CrossRef] [PubMed]

34. Brindel, P.; Doyon, F.; Rachédi, F.; Boissin, J.-L.; Sebbag, J.; Shan, L.; Chungue, V.; Sun, L.Y.K.; Bost-Bezeaud, F.; Petitdidier, P. Menstrual and reproductive factors in the risk of differentiated thyroid carcinoma in native women in French Polynesia: A population-based case-control study. Am. J. Epidemiol. 2008, 167, 219-229. [CrossRef]

35. Adami, G.; Saag, K.G. Osteoporosis pathophysiology, epidemiology, and screening in rheumatoid arthritis. Curr. Rheumatol. Rep. 2019, 21, 34. [CrossRef] [PubMed]

36. Oh, C.-M.; Kong, H.-J.; Kim, E.; Kim, H.; Jung, K.-W.; Park, S.; Won, Y.-J. National epidemiologic survey of thyroid cancer (NEST) in Korea. Epidemiol. Health 2018, 40, e2018052. [CrossRef] 